EPJ Web of Conferences 71, 00119 (2014)

DOI: $10.1051 /$ epjconf/20147100119

(C) Owned by the authors, published by EDP Sciences, 2014

\title{
Hadrosynthesis and Quark Confinement
}

\author{
Helmut Satz $z^{1, a}$ \\ ${ }^{1}$ Fakultät für Physik, Universität Bielefeld, D-33501 Bielefeld, Germany
}

\begin{abstract}
Multihadron production in high energy collisions, from $e^{+} e^{-}$annihilation to heavy ion interactions, shows remarkable thermal behaviour, specified by a universal "Hagedorn" temperature. We argue that this hadronic radiation is formed by tunnelling through the event horizon of colour confinement, i.e., that it is the QCD counterpart of Hawking-Unruh radiation from black holes. It is shown to be emitted at a universal temperature $T_{H} \simeq(\sigma / 2 \pi)^{1 / 2}$, where $\sigma$ denotes the string tension. Since the event horizon does not allow information transfer, the radiation is thermal "at birth".
\end{abstract}

\section{Introduction}

The pattern of multihadron production in high energy collisions is one of the most fascinating topics in strong interaction physics. In particular, the origin of the universal thermal behaviour observed in this production has both interested and puzzled physicists for many years. We discuss here a novel approach to this subject, based on the analogy between gravitational confinement in black holes and colour confinement in hadronic systems [1]. This approach is all the more interesting since it has been shown to provide an essentially parameter-free description of a vast range of hadron production data [2].

Quantum chromodynamics, through colour confinement, restricts quarks and gluons to move in a limited region of space. In a black hole, matter and light are confined by gravitation to remain within a restricted region of space. This similarity was noted quite soon after the advent of QCD, and it was suggested that hadrons were the analogue of black holes in strong interaction physics [3-5]. In the case of gravitational black holes, the isolation of the system is not quite absolute: in the strong field at the outer edge, quantum excitation can lead to the emission of Hawking radiation into the physical vacuum [6]. Since no information transfer between the inside and the outside of the black hole is allowed, this radiation must give equal a priori weights to all possible states on the outside, and hence it is thermal at the point of formation. In this report, based on joint work with Paolo Castorina and Dmitri Kharzeev [1], we want to show that high energy collisions in strong interaction physics produce a self-similar cascade of "white holes" (colourless from the outside, but coloured inside), and that the Hawking radiation arising at the confinement horizon of these can provide the thermal behaviour observed quite universally in all soft hadron production $[7,8]$.

We begin by recalling briefly the relevant basic aspects of black hole physics and indicate some first relations to strong interactions. We then turn to high energy $e^{+} e^{-}$annihilation as the simplest

\footnotetext{
ae-mail: satz@physik.uni-bielefeld.de
} 
hadron production process and study the effect of quark-antiquark pair excitation and string breaking. The resulting scenario is readily generatlized to hadronic interactions. Finally, we comment on stochastic vs. kinetic thermalization.

\section{Black Holes and Hawking Radiation}

A black hole is the final stage of a neutron star after gravitational collapse [9]. It has a mass $M$ concentrated in such a small volume that the resulting gravitational field confines all matter and even photons to remain inside the event horizon $R$ of the system: no causal connection with the outside is possible. As a consequence, black holes have three (and only three) observable properties: mass $M$, charge $Q$ and angular momentum $J$. This section will address mainly black holes with $Q=J=0$; we shall come back to the more general properties in section 4 .

Classically, a black hole would persist forever and remain forever invisible. On a quantum level, however, its constituents (photons, leptons and hadrons) have a non-vanishing chance to escape by tunnelling through the barrier presented by the event horizon. The resulting Hawking radiation [6] cannot convey any information about the internal state of the black hole; it must be therefore be thermal, and it was shown that for a non-rotating black hole of vanishing charge (denoted as Schwarzschild black hole), the corresponding radiation temperature is

$$
T_{B H}=\frac{1}{8 \pi G M}
$$

where $G$ is the gravitational constant. This temperature is inversely proportional to the mass of the black hole, and since the radiation reduces the mass, the radiation temperature will increase with time. For black holes of stellar size, however, one finds $T_{B H} \lesssim 2 \times 10^{-8}{ }^{\circ} \mathrm{K}$. This is many orders of magnitude below the $2.7^{\circ} \mathrm{K}$ cosmic microwave background, and hence not detectable.

In general relativity, the event horizon arises as consequence of the Schwarzschild metric and its generalizations to $Q \neq 0, J \neq 0$. The occurrence and role of the event horizon for thermal radiation was subsequently generalized by Unruh [10]. A system undergoing uniform acceleration $a$ relative to a stationary observer eventually reaches a classical turning point and thus encounters an event horizon. Let us recall the resulting hyperbolic motion [11]. A point mass $m$ subject to a constant force $F$ satisfies the equation of motion

$$
\frac{d}{d t} \frac{m v}{\sqrt{1-v^{2}}}=F
$$

where $v(t)=d x / d t$ is the velocity, normalized to the speed of light $c=1$. This equation is solved by the parametric form

$$
x=\frac{1}{a} \cosh a \tau \quad t=\frac{1}{a} \sinh a \tau,
$$

where $a=F / m$ denotes the acceleration in the instantaneous rest frame of $m$, and $\tau$ the proper time, with $d \tau=\sqrt{1-v^{2}} d t$.

The resulting world line is shown in Fig. 1. It corresponds to the mass $m$ coming from $x=\infty$ at $t=-\infty$ at with a velocity arbitrarily close to that of light, decelerating uniformly until it comes to rest at the classsical turning point $x_{H}=-(1 / a)$. It then accelerates again and returns to $x=\infty$ at $t=\infty$, approaching the speed of light. For given $a, x_{H}$ is thus the event horizon beyond which $m$ classically cannot pass. The only signal an observer can detect of the passage of $m$ is thermal quantum radiation of temperature $[6,10,12,13]$

$$
T_{U}=\frac{a}{2 \pi} .
$$




\section{ICNFP 2013}

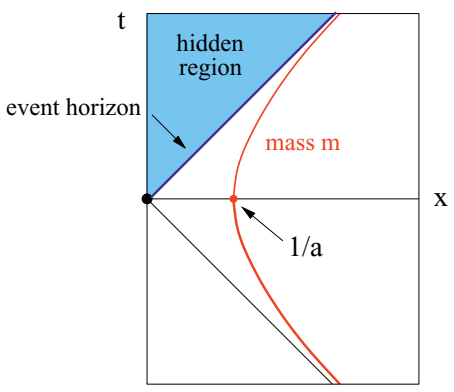

Figure 1. Hyperbolic motion

In the case of gravity, we have the force

$$
F=m a=G \frac{m M}{R^{2}}
$$

on a probe of mass $m$. From the dependence of the gravitational metric on black hole mass and radius, we obtain

$$
R_{g}=2 G M
$$

for the Schwarzschild radius of the black hole, leading to $a=1 /(4 G M)$ for the acceleration at the event horizon and thus back to eq. (1). We also see why the temperature of Hawking radiation decreases with increasing black hole mass: since $R_{g}$ grows linearly with $M$, increasing the black hole mass decreases its energy density and hence the Hawking temperature $T_{B H}$. $\mathrm{GeV}$ :

It is instructive to consider the Schwarzschild radius of a typical hadron, assuming a mass $m \sim 1$

$$
R_{g}^{\text {had }} \simeq 1.3 \times 10^{-38} \mathrm{GeV}^{-1} \simeq 2.7 \times 10^{-39} \mathrm{fm} .
$$

To become a gravitational black hole, the mass of the hadron would thus have to be compressed into a volume more than $10^{100}$ times smaller than its actual volume, with a radius of about $1 \mathrm{fm}$. On the other hand, if instead we increase the interaction strength from gravitation to strong interaction [3], we gain in the resulting "strong" Schwarzschild radius $R_{s}^{\text {had }}$ a factor

$$
\frac{\alpha_{s}}{G m^{2}}
$$

where $\alpha_{s}$ is the dimensionless strong coupling and $G m^{2}$ the corresponding dimensionless gravitational coupling for the case in question. This leads to

$$
R_{s}^{h a d} \simeq \frac{2 \alpha_{s}}{m}
$$

which for $\alpha_{s} \simeq 2.5$ gives $R_{s}^{\text {had }}=1 \mathrm{fm}$. In other words, the confinement radius of a hadron is about the size of its "strong" Schwarzschild radius, so that we could consider quark confinement as the strong interaction version of the gravitational confinement in black holes $[3,4]$.

The event horizon of a black hole arises from the deformation of the Minkowski metric due to the strong gravitational force. Such a geometrization of force effects has also been employed in non-linear electrodynamics, where it can lead to similar spatial constraints [14]. A corresponding extension to QCD is discussed in [1]; we shall here concentrate on the more phenomenological aspects. 


\section{Pair Production and String Breaking}

In the previous section, we had considered constituents of a black hole undergoing accelerated motion in classical space-time. In this section, we shall first address the modifications which arise if the underlying space-time manifold is specified by quantum field theory, so that in the presence of a strong field the vacuum becomes unstable under pair production. Next we turn to the specific additional features which come in when the basic constituents are subject to colour confinement and can only exist in colour neutral bound states.

As basic starting point, we consider two-jet $e^{+} e^{-}$annihilation at cms energy $\sqrt{s}$,

$$
e^{+} e^{-} \rightarrow \gamma \rightarrow q \bar{q} \rightarrow \text { hadrons. }
$$

The initially produced $q \bar{q}$ pair flies apart, subject to the constant confining force given by the string tension $\sigma$; this results in hyperbolic motion [15-17] of the type discussed in the previous section. At $t=0$, the $q$ and $\bar{q}$ separate with an initial velocity $v_{0}=p / \sqrt{p^{2}+m^{2}}$, where $p \simeq \sqrt{s} / 2$ is the momentum of the primary constituents in the overall $\mathrm{cms}$ and $m$ the effective quark mass. We now have to solve Eq. (2) with this situation as boundary condition; the force

$$
F=\sigma,
$$

is given by the string tension $\sigma$ binding the $q \bar{q}$ system. The solution is

$$
\tilde{x}=\left[1-\sqrt{1-v_{0} \tilde{t}+\tilde{t}^{2}}\right]
$$

with $\tilde{x}=x / x_{0}$ and $\tilde{t}=t / x_{0}$; here the scale factor

$$
x_{0}=\frac{m}{\sigma} \frac{1}{\sqrt{1-v_{0}^{2}}}=\frac{1}{a} \gamma
$$

is the inverse of the acceleration $a$ measured in the overall $\mathrm{cms}$. The velocity becomes

$$
v(t)=\frac{d x}{d t}=\frac{\left(v_{0} / 2\right)-\tilde{t}}{\sqrt{1-v_{0} \tilde{t}+\tilde{t}^{2}}} ;
$$

it vanishes for

$$
\tilde{t}^{*}=\frac{v_{0}}{2} \Rightarrow t^{*}=\frac{v_{0}}{2} \frac{m}{\sigma} \gamma
$$

thus defining

$$
x\left(t^{*}\right)=\frac{m}{\sigma} \gamma\left(1-\sqrt{1-\left(v_{0}^{2} / 4\right)}\right) \simeq \frac{\sqrt{s}}{2 \sigma}
$$

as classical turning point and hence as the classical event horizon measured in the overall $\mathrm{cms}$ (see Fig. 2).

Eq. (16) allows the $q$ and the $\bar{q}$ to separate arbitrarily far, provided the pair has enough initial energy; this clearly violates colour confinement. Our mistake was to consider the $q \bar{q}$ system in a classical vacuum; in quantum field theory, the vacuum itself contains virtual $q \bar{q}$ pairs, and hence it is not possible to increase the potential energy of a given $q \bar{q}$ state beyond the threshold value necessary to bring such a $q \bar{q}$ pair on-shell. In QED, the corresponding phenomenon was addressed by Schwinger [18], who showed that in the presence of a constant electric field of strength $\mathcal{E}$ the probability of producing an electron-positron pair is given by

$$
P(M, \mathcal{E}) \sim \exp \left\{-\pi m_{e}^{2} / e \mathcal{E}\right\},
$$




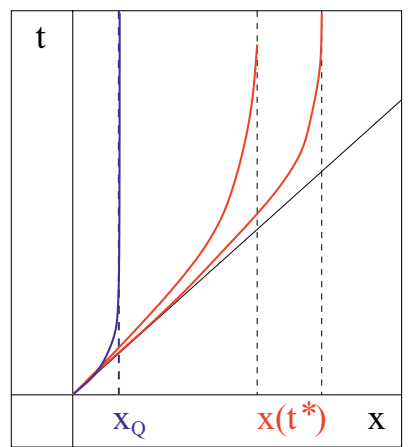

Figure 2. Classical and quantum horizons in $q \bar{q}$ separation

with $m_{e}$ denoting the electron mass and $e$ denoting the electric charge. In QCD, we expect a similar effect when the string tension exceeds the pair production limit, i.e., when

$$
\sigma x>2 m
$$

where $m$ specifies the effective quark mass. Beyond this point, any further stretching of the string is expected to produce a $q \bar{q}$ pair with the probability

$$
P(M, \sigma) \sim \exp \left\{-\pi m^{2} / \sigma\right\}
$$

with the string tension $\sigma$ replacing the electric field strength $e \mathcal{E}$. This string breaking acts like a quantum event horizon $x_{q}=2 \mathrm{~m} / \sigma$, which becomes operative long before the classical turning point is ever reached (see Fig. 2). Moreover, the resulting allowed separation distance for our $q \bar{q}$ pair, the colour confinement radius $x_{Q}$, does not depend on the initial energy of the primary quarks.

There are some important differences between QCD and QED. In case of the latter, the initial electric charges which lead to the field $\mathcal{E}$ can exist independently in the physical vacuum, and the produced pair can be simply ionized into an $e^{+}$and an $e^{-}$. In contrast, neither the primary quark nor the constituents of the $q \bar{q}$ pair have an independent existence, so that in string breaking colour neutrality must be preserved. As a result, the Hawking radiation in QCD must consist of $q \bar{q}$ pairs, and these can be produced in an infinite number of different excitation states of increasing mass and degeneracy. Moreover, the $q \bar{q}$ pair spectrum is itself determined by the strength $\sigma$ of the field, in contrast to the exponent $m_{e}^{2} / \mathcal{E}$ in eq. (17), where the value of $\mathcal{E}$ has no relation to the electron mass $m_{e}$.

Hadron production in $e^{+} e^{-}$annihilation is believed to proceed in the form of a self-similar cascade $[19,20]$. Initially, we have the separating primary $q \bar{q}$ pair,

$$
\gamma \rightarrow[q \bar{q}]
$$

where the square brackets indicate colour neutrality. When the energy of the resulting color flux tube becomes large enough, a further pair $q_{1} \bar{q}_{1}$ is excited from the vacuum,

$$
\gamma \rightarrow\left[q\left[\bar{q}_{1} q_{1}\right] \bar{q}\right]
$$

Although the new pair is at rest in the overall $\mathrm{cms}$, each of its constituents has a transverse momentum $k_{T}$ determined, through the uncertainty relation, by the transverse dimension $r_{T}$ of the flux tube. The 
slow $\bar{q}_{1}$ now screens the fast primary $q$ from its original partner $\bar{q}$, with an analoguous effect for the $q_{1}$ and the primary antiquark. To estimate the $q \bar{q}$ separation distance at the point of pair production, we recall that the basic thickness of the flux tube connecting the $q \bar{q}$ pair is in string theory given by [21]

$$
r_{T}=\sqrt{\frac{2}{\pi \sigma}}
$$

higher excitations lead to a greater thickness and eventually to a divergence (the "roughening" transition). From the uncertainty relation we then have

$$
k_{T} \simeq \sqrt{\frac{\pi \sigma}{2}} .
$$

With this transverse energy is included in eq. (18), we obtain for the pair production separation $x_{Q}$

$$
\sigma x_{q}=2 \sqrt{m^{2}+k_{T}^{2}} \Rightarrow x_{q} \simeq \frac{2}{\sigma} \sqrt{m^{2}+(\pi \sigma / 2)} \simeq \sqrt{\frac{2 \pi}{\sigma}} \simeq 1.1 \mathrm{fm}
$$

with $\sigma=0.2 \mathrm{GeV}^{2}$ and $m^{2} \ll \sigma$.

Once the new pair is present, we have a colour-neutral system $q \bar{q}_{1} q_{1} \bar{q}$; but since there is a sequence of connecting string potentials $q \bar{q}_{1}, \bar{q}_{1} q_{1}$ and $q_{1} \bar{q}$, the primary string is not yet broken. To achieve that, the binding of the new pair has to be overcome, i.e., the $q_{1}$ has to tunnel through the barrier of the confining potential provided by $\bar{q}_{1}$, and vice versa. Now the $q$ excerts a longitudinal force on the $\bar{q}_{1}$, the $\bar{q}$ on the $q_{1}$, resulting in a longitudinal acceleration and ordering of $q_{1}$ and $\bar{q}_{1}$. When (see Fig. 3)

$$
\sigma x\left(q_{1} \bar{q}_{1}\right)=2 \sqrt{m^{2}+k_{T}^{2}},
$$

the $\bar{q}_{1}$ reaches its $q_{1} \bar{q}_{1}$ horizon; on the other hand, when

$$
\sigma x\left(q \bar{q}_{1}\right)=2 \sqrt{m^{2}+k_{T}^{2}},
$$

the new flux tube $q \bar{q}_{1}$ reaches the energy needed to produce a further pair $q_{2} \bar{q}_{2}$. The $\bar{q}_{2}$ screens the primary $q$ from the $q_{1}$ and forms a new flux tube $q \bar{q}_{2}$. At this point, the original string is broken, and the remaining pair $\bar{q}_{1} q_{2}$ form a colour neutral bound state which is emitted as Hawking radiation in the form of hadrons, with the relative weights of the different states governed by the corresponding Unruh temperature. The resulting pattern is schematically illustrated in Fig. 3.

To determine the temperature of the hadronic Hawking radiation, we return to the original pair excitation process. To produce a quark of momentum $k_{T}$, we have to bring it on-shell and change its velocity from zero to $v=k_{T} /\left(m^{2}+k_{T}^{2}\right)^{1 / 2} \simeq 1$. This has to be achieved in the time of the fluctuation determined by the virtuality of the pair, $\Delta \tau=1 / \Delta E \simeq 1 / 2 k_{T}$. The resulting acceleration thus becomes

$$
a=\frac{\Delta v}{\Delta \tau} \simeq 2 k_{T} \simeq \sqrt{2 \pi \sigma} \simeq 1.1 \mathrm{GeV}
$$

which leads to

$$
T_{H}=\frac{a}{2 \pi} \simeq \sqrt{\frac{\sigma}{2 \pi}} \simeq 180 \mathrm{MeV}
$$

for the hadronic Unruh temperature. It governs the momentum distribution and the relative species abundances of the emitted hadrons. 


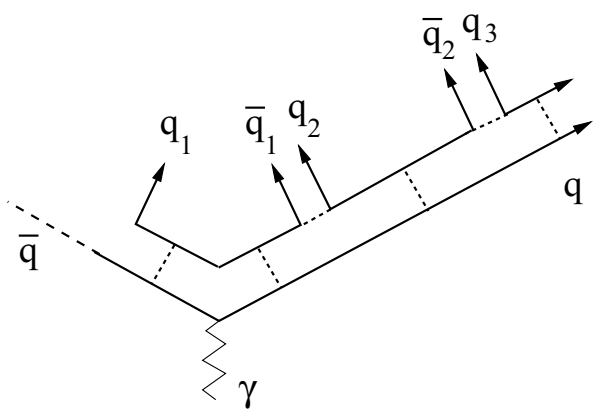

Figure 3. String breaking through $q \bar{q}$ pair production

A given step in the evolution of the hadronization cascade of a primary quark or antiquark produced in $e^{+} e^{-}$annihilation thus involves several distinct phenomena. The color field created by the separating $q$ and $\bar{q}$ produces a further pair $q_{1} \bar{q}_{1}$ and then provides an acceleration of the $q_{1}$, increasing its longitudinal momentum. When it reaches the $q_{1} \bar{q}_{1}$ confinement horizon, still another pair $q_{2} \bar{q}_{2}$ is excited; the state $\bar{q}_{1} q_{2}$ is emitted as a hadron, the $\bar{q}_{2}$ forms together with the primary $q$ a new flux tube. This pattern thus step by step increases the longitudinal momentum of the "accompanying" $\bar{q}_{i}$ as well as of the emitted hadron. This, together with the energy of the produced pairs, causes a corresponding deceleration of the primary quarks $q$ and $\bar{q}$, in order to maintain overall energy conservation. In Fig. 4 , we show the world lines given by the acceleration $\bar{q}_{i} \rightarrow \bar{q}_{i+1}\left(q_{i} \rightarrow q_{i+1}\right)$ and that of formation threshold of the hadrons $\bar{q}_{i} q_{i+1}$ and the corresponding antiparticles.

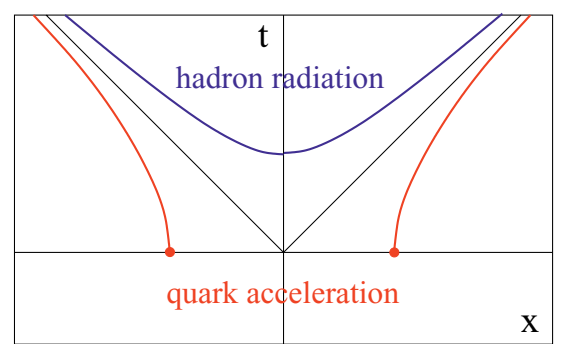

Figure 4. Quark acceleration and hadronization world lines

The energy loss and deceleration of the primary quark $q$ in this self-similar cascade, together with the acceleration of the accompanying partner $\bar{q}_{i}$ from the successive pairs brings $q$ and $\bar{q}_{i}$ closer and closer to each other in momentum, from an initial separation $q \bar{q}_{1}$ of $\sqrt{s} / 2$, until they finally are combined into a hadron and the cascade is ended. The resulting pattern is shown in Fig. 5.

The number of emitted hadrons, the multiplicity $v(s)$, follows quite naturally from the picture presented here. The classical string length, in the absence of quantum pair formation, is given by the classical turning point determined in eq. (16). The thickness of a flux tube of such an "overstretched" string is known [21]; instead of eq. (22) we have

$$
R_{T}^{2}=\frac{2}{\pi \sigma} \sum_{k=0}^{K} \frac{1}{2 k+1} \simeq \frac{2}{\pi \sigma} \ln 2 K,
$$




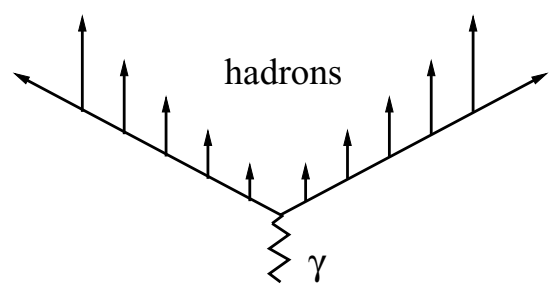

Figure 5. Hadronization in $e^{+} e^{-}$annihilation

where $K$ is the string length in units of an intrinsic string vibration measure. From eq. (16) we thus get

$$
R_{T}^{2}=\simeq \frac{2}{\pi \sigma} \ln \sqrt{s}
$$

for the flux tube thickness in the case of the classical string length. Because of pair production, this string breaks whenever it is stretched to the length $x_{q}$ given in eq. (24); its thickness $r_{T}$ at this point is given by eq. (22). The multiplicity can thus be estimated by the ratio of the corresponding classical to quantum transverse flux tube areas,

$$
v(s) \sim \frac{R_{T}^{2}}{r_{T}^{2}} \simeq \ln \sqrt{s},
$$

and is found to grow logarithmically with the $e^{+} e^{-}$annihilation energy, as is in fact observed experimentally.

So far, we have considered the general nature of hadroproduction in electron-positron annihilation, as it arises in the framework of Unruh radiation. To show that this can really account for the actual data, a quantitative analysis of production data over the total available range of annihilation energies has to be performed, and in particular, the non-vanishing strange quark mass has to be taken into account. Such an analysis was carried out in [2], and it is found to lead to excellent agreement with all the data. The formalism contains only two parameters, the string tension $\sigma$ and the strange quark mass $m_{s}$. Since both are determined from other, independent measurements, we conclude that the Unruh scheme provides as parameter-free explanation of hadroproduction in electron-positron annihilation.

\section{Stochastic vs. Kinetic Thermalization}

In statistical mechanics, a basic topic is the evolution of a system of many degrees of freedom from non-equilibrium to equilibrium. Starting from a non-equilibrium initial state of low entropy, the system is assumed to evolve as a function of time through collisions to a time-independent equlibrium state of maximum entropy. In other words, the system loses the information about its initial state through a sequence of collisions and thus becomes thermalized. In this sense, thermalization in heavy ion collisions was studied as the transition from an initial state of two colliding beams of "parallel" partons to a final state in which these partons have locally isostropic distributions. This "kinetic" thermalization requires a sufficient density of constituents, sufficiently large interaction cross sections, and a certain amount of time.

From such a point of view, the observation of thermal hadron production in high energy collisions in general, in $e^{+} e^{-}$and $p p$ interactions as well as in heavy ion collisions, is a puzzle: how could these systems ever "have reached" thermalization? Already Hagedorn had therefore concluded that the emitted hadrons were "born in equilibrium" [22]. 
Hawking radiation provides a stochastic rather than kinetic approach to equilibrium. The barrier to information transfer provided by the event horizon requires that the resulting radiation states excited from the vacuum are distributed according to maximum entropy, with a temperature determined by the strength of the "confining" field. The ensemble of all produced hadrons, averaged over all events, then leads to the same equilibrium distribution as obtained in hadronic matter by kinetic equilibration. In the case of a very high energy collision with a high average multiplicity already one event can provide such equilibrium; because of the interruption of information transfer at each of the successive quantum colour horizons, there is no phase relation between two successive production steps in a given event. The destruction of memory, which in kinetic equilibration is achieved through sufficiently many successive collisions, is here automatically provided by the tunnelling process.

So the thermal hadronic final state in high energy collisions is not reached through a kinetic process; it is rather provided by successively throwing dice.

\section{Conclusions}

We have shown that quantum tunnelling through the colour confinement horizon leads to thermal hadron production in the form of Hawking radiation. In particular, this implies:

- The radiation temperature $T_{H}$ is determined by the transverse extension of the colour flux tube, giving

$$
T_{H}=\sqrt{\frac{\sigma}{2 \pi}}
$$

in terms of the string tension $\sigma$.

- The multiplicity $v(s)$ of the produced hadrons is determined by the increase of the flux tube thickness with string length, leading to

$$
v(s) \simeq \ln \sqrt{s},
$$

where $\sqrt{s}$ denotes the collision energy.

- In statistical QCD, thermal equilibrium is reached kinetically from an initial non-equilibrium state, with memory destruction through successive interactions of the constituents. In high energy collisions, tunnelling prohibits information transfer and hence leads to stochastic production, so that we have a thermal distribution from the outset.

\section{References}

[1] P. Castorina, D. Kharzeev and H. Satz, Europ. Phys. J. C52 187 (2007).

[2] F. Becattini et al., Europ. Phys. J. CC 56493 (2008).

[3] E. Recami and P. Castorina, Lett. Nuovo Cim. 15347 (1976).

[4] A. Salam and J. Strathdee, Phys. Rev. D 184480 (1978).

[5] A. F. Grillo and Y. Srivastava, Phys. Lett. B 85377 (1979).

[6] S. W. Hawking, Comm. Math. Phys. 43199 (1975).

[7] R. Hagedorn, Nuovo Cim. Suppl. 3147 (1965); Nuovo Cim. A 561027 (1968).

[8] F. Becattini, Z. Phys. C 69485 (1996) $\left(e^{+} e^{-}\right)$;

F. Becattini and U. Heinz, Z. Phys. C 76268 (1997) ( $p p / p \bar{p})$;

J. Cleymans and H. Satz, Z. Phys. C 57135 (1993) (heavy ions);

F. Becattini et al., Phys. Rev. C64 (2001) 024901 (heavy ions);

P. Braun-Munziger, K. Redlich and J. Stachel, in Quark-Gluon Plasma 3, R. C. Hwa and X.-N. Wang (Eds.), World Scientific, Singapore 2003 (heavy ions). 
[9] See e.g., Li Zhi Fang and R. Ruffini, Basic Concepts in Relativistic Astrophysics, World Scientific, Singapore 1983.

[10] W. G. Unruh, Phys. Rev. D 14870 (1976).

[11] For a clear discussion and references to the original solutions by M. Born (1909) and A. Sommerfeld (1910),, see W. Pauli, Relativitätstheorie, in Enzyklopädie der mathematischen Wissenschaften, Teubner Verlag, Leipzig 1921; English version Theory of Relativity, Pergamon Press, 1958.

[12] T. D. Lee, Nucl. Phys. B 264437 (1986).

[13] M. K. Parikh and F. Wilczek, Phys. Rev. Lett. 855042 (2000).

[14] M. Novello et al., Phys. Rev. D 61045001 (2000).

[15] S. Barshay and W. Troost, Phys. Lett. 73B 437 (1978).

[16] A. Hosoya, Progr. Theoret. Phys. 61280 (1979).

[17] M. Horibe, Progr. Theoret. Phys. 61661 (1979).

[18] J. Schwinger, Phys. Rev. 82664 (1951).

[19] J. D. Bjorken, Lecture Notes in Physics (Springer) 5693 (1976).

[20] A. Casher, H. Neuberger and S. Nussinov, Phys. Rev. D 20179 (1979).

[21] M. Lüscher, G. Münster and P. Weisz, Nucl. Phys. B 1801 (1981).

[22] R. Hagedorn, Thermodynamics of Strong Interactions, CERN 71-12 (1971);

R. Stock, Phys. Lett. B 456277 (1999). 